\title{
Erratum: Optical signatures of electron-phonon decoupling due to strong light-matter interactions [Phys. Rev. B 102, 235303 (2020)]
}

\author{
Emil V. Denning (), Matias Bundgaard-Nielsen, and Jesper Mørk
}

(Received 9 February 2021; published 24 February 2021)

DOI: 10.1103/PhysRevB.103.079903

We have become aware of a number of errors in the published paper. These errors do not concern the main results of the paper and only lead to minor quantitative corrections to some of the presented calculations. For completeness, we hereby point out the errors and present the corrected form of equations that contain errors as well as any figures that are influenced by these errors.

The expression for $C_{Z Z}(\tau)$ in Eq. (D3) on p. 8 should read

$$
C_{Z Z}(\tau)=\int_{0}^{\infty} d \nu J(\nu)[1-F(\nu)]^{2}[\operatorname{coth}(\beta \hbar \nu / 2) \cos (\nu \tau)-i \sin (\nu \tau)]
$$

This error in $C_{Z Z}(\tau)$ was also carried over to Eq. (D6) on p. 8, where $\epsilon_{Z Z}$ should read

$$
\epsilon_{Z Z}=\frac{\pi}{2} J\left(2 g_{\mathrm{v}}\right)\left[1-F\left(2 g_{\mathrm{v}}\right)\right]^{2} \text {. }
$$

This is also the case for the approximate expression for $\Gamma_{A}$, which appears below Eq. (D6) on p. 8 and in Eq. (6) on p. 5. The correct approximate expression for $\Gamma_{A}$ is

$$
\Gamma_{A} \simeq \frac{\pi}{2} J\left(2 g_{\mathrm{v}}\right)\left[1-F\left(2 g_{\mathrm{v}}\right)\right]^{2} .
$$

The expression for $\epsilon_{Z Z}$ was used in Fig. 5 on p. 5 and Fig. 7 on p. 8. Below, we provide these figures calculated with the error in $\epsilon_{Z Z}$ corrected in Figs. 1 and 2.

In Eq. (D3) on p. 8 there is a factor of $\hbar$ missing in the expression of $C_{Y Z}(\tau)$, which should read

$$
C_{Y Z}(\tau)=-B_{\mathrm{v}} \int_{0}^{\infty} d \nu J(v) v^{-1} F(\nu)[1-F(\nu)][i \cos (\nu \tau)+\operatorname{coth}(\beta \hbar \nu / 2) \sin (\nu \tau)] .
$$

This is a typographical error, which has not been carried over to any other results or equations.

There is an error in the definitions of $B_{\mathrm{v}}, B_{X}$, and $B_{Y}$, which below Eq. (4) on p. 3, below Eq. (C1), and below Eq. (C2) on p. 7. The correct definitions are

$$
B_{\mathrm{v}}=\left\langle e^{ \pm V^{\prime}}\right\rangle, \quad B_{X}=\left(e^{V^{\prime}}+e^{-V^{\prime}}-2\right) / 2, \quad B_{Y}=i\left(e^{V^{\prime}}-e^{-V^{\prime}}\right) / 2,
$$

where $V^{\prime}=\sum_{\mathbf{k}} \frac{f_{\mathbf{k}}}{\nu_{\mathbf{k}}}\left(b_{\mathbf{k}}^{\dagger}-b_{\mathbf{k}}\right)$. These errors have not been carried over to other equations or any results presented in the article.

The definition of $Y$ below Eq. (C2) has a sign error and should read

$$
Y=-i g\left(|X\rangle\langle 0|a-| 0\rangle\langle X| a^{\dagger}\right) .
$$

This error has not influenced other equations or any results presented in the article.

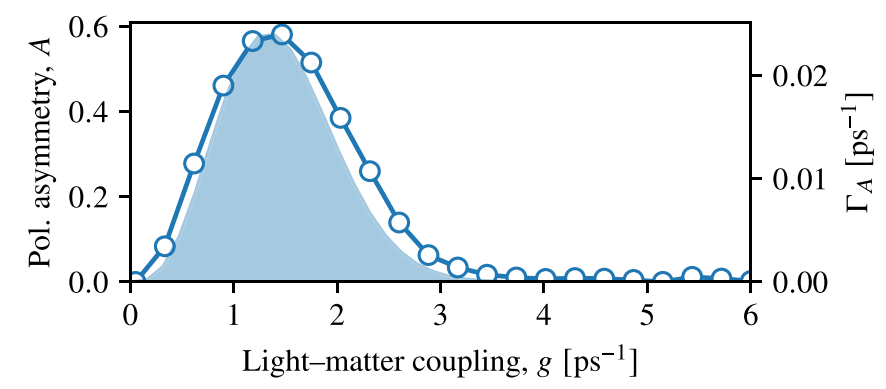

FIG. 1. Corrected version of Fig. 5. 
(a)

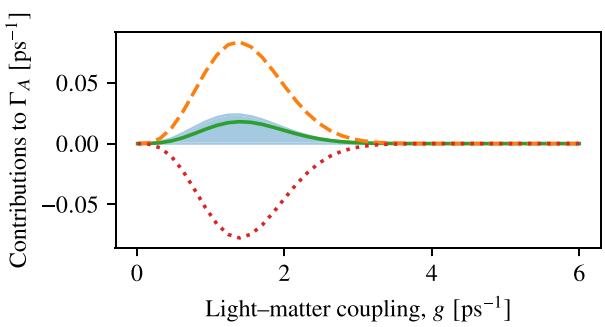

(b)

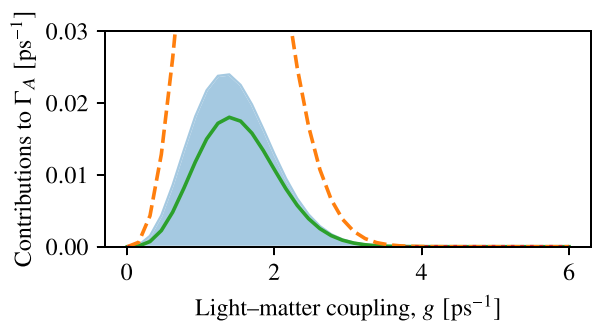

FIG. 2. (a) Corrected version of Fig. 7. (b) Zoom in of panel a. 